\title{
Histopathological Analysis of Arctium Lappa Extract in the Treatment of Cutaneous Melanoma in C57BL/6 Mice
}

\author{
Souza $S \mathrm{M}^{1}$, Araújo $\mathrm{M} \mathrm{F}^{1}$, Silva A $\mathrm{A}^{2}$, Moreira $\mathrm{M} \mathrm{R} \mathrm{A}{ }^{3}$, Zoccal $\mathrm{K} \mathrm{F}^{\mathbf{1}}$ and Tefé Silva $\mathrm{C}^{1 *}$ \\ ${ }^{1}$ Centro Universitário Barão de Mauá, Ribeirão Preto, Brazil \\ ${ }^{2}$ Department of Physiology, University of Mississipi Medical Center, Jackson, MS, United States of America \\ ${ }^{3}$ Department of Clinical, Toxicological and Bromatological Analyzes. Faculty of Pharmaceutical Sciences of Ribeirão Preto, University of \\ São Paulo (FCFRP-USP), Ribeirão Preto, Brazil \\ *Corresponding author: Cristiane Tefé- Silva, Department of Clinical, Toxicological and Bromatological Analyzes. Faculty of \\ Pharmaceutical Sciences of Ribeirão Preto, University of São Paulo (FCFRP-USP), Ribeirão Preto, Brazil
}

\section{ARTICLE INFO}

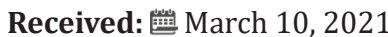

Published: 蔧 March 18, 2021

Citation: Souza S M, Araújo M F, Silva A A, Moreira M R A, Zoccal K F, Tefé Silva C. Green Synthesis of Phytochemical Nanoparticles and Their Antimicrobial Activity, A Review Study. Biomed J Sci \& Tech Res 34(4)-2021. BJSTR. MS.ID.005581.

Keywords: Skin Cancer; Melanoma; Alternative Plants; Arctinin; Burdock

\section{ABSTRACT}

Background: Melanoma is a very aggressive skin cancer responsible for thousands of deaths annually. Due to its impact on public health, many recent studies have sought to unravel novel, more effective alternatives for treatment melanoma. Previous studies showed that Arctium lappa, popularly known as burdock, contains lignans and may exert cytotoxic specific effects against cancerous cells including prostate hyperplasia, leukemia, and certain cellular lineages of pancreatic cancer. In the present study, we evaluated $A$. lappa potential actions as an alternative cutaneous melanoma treatment in C57BL/6 mice. Methods. Primary tumor studies were performed using B16F10 melanoma cells injected subcutaneously into the dorsal superior region of the mice. $A$. lappa hydroalcoholic extracts were injected every 5 days along the course of a 25-day treatment regimen protocol. Vehicle-treated control mice were submitted to the same conditions. Results. We observed that A. lappa extract administration for 25 days reduced tumorigenesis, angiogenesis and mitotic index when compared to the vehicle-treated group. Conclusion. Altogether, these results provide evidence that A. lappa contributes to improved host response against melanoma progression and that it may be an effective alternative approach to the treatment of cutaneous melanoma.

\section{Background}

Melanoma is a very aggressive form of skin cancer in humans with a multifactorial etiology, resulting from interactions between environmental, genetic and host factors. It affects both men and women, and it is more common in Caucasians due to their greater sensitivity to sun exposure [1,2]. The incidence of cutaneous melanoma has grown in recent years [3-5] with a tendency to duplicate every 10-20 years [4]. Recent studies show that the highest mortality rates from melanoma without distinction of sex were in countries of Eastern Europe, Oceania and United States [6]. While early stage-diagnosed disease is effectively treated with surgical removal, metastatic stage is almost universally fatal and good clinical response rate to chemotherapy is less than $30 \%[1,2]$.
Melanoma originates from epidermal melanocytes, the melaninproducing cells, and involves environmental and genetic factors. Exposure to sunlight is the primary environmental risk factor associated with development of melanoma [5], with ultraviolet (UV) radiation being the main triggering factor for its development due to UV radiation ability to break down chromosomes and promote changes in cellular DNA. During prolonged exposure to UV light, keratinocytes and/or melanocytes may undergo signaling pathway mutations related to cell survival and proliferation as the initial step for development of skin cancer $[7,8]$. Several biological changes are involved in development and progression of cancers such as: deregulated cell growth, angiogenesis, activation of oncogenes (BRAF gen, with has the highest prevalence mutation 
in melanoma) [9], inactivation of tumor suppressing genes (e.g., cyclin-dependent kinase inhibitor 2A (CDKN2A) (p16), a gene that is mutated in autosomal dominant familial melanomas [10], and loss of PTEN (phosphatase and tensin homolog) gene. In addition, antiapoptotic signals are activated and neoplasic cells develop the capacity to undergo metastasis. Recently, other processes have been implicated in the development of cancer including immune system evasion, genomic instability, and alterations in cellular metabolism.

Thus, due to melanoma's significant impact on public health many studies have looked for more effective alternatives to treat melanoma. The Arctium lappa, popularly known as burdock, is a plant from the Asteraceae family of European origin [11]. Due to the presence of lignans arctigenin and arctinin, which have anticarcinogenic and anti-tumorigenic properties, extracts from $A$. lappa seeds 11 have been suggested as potential alternative against cancer. Lignans have been reported to increase sensitivity of tumor cells to chemotherapeutic drugs [12], and to exert cytotoxic effects inducing apoptosis of tumor cells by reducing cells' ability to utilize glucose with consequent impairment of energy production $[5,13,14]$. Previous reports also suggest anti-cancer properties of A. lappa in prostatic hyperplasia, leukemia and pancreatic cancer [15-17]. In face of A. lappa potential antitumorigenic activity and the difficulties in developing better anti-melanoma therapies, in the present study we examined the effects of burdock extract as a novel alternative therapy to attenuate development and progression of experimentally induced melanoma in C57BL/6 mice. For this purpose, we took advantage of B16F10 melanoma cells, are a well-validated model for primary tumor, to induce subcutaneous tumors. We found that chronic treatment with A. lappa extract significantly reduced tumorigenesis, angiogenesis and mitotic index of cutaneous melanoma in C57BL/6 mice.

\section{Methods}

\section{Animal Care}

Female C57BL/6 mice (6-8 weeks old) were maintained at our animal facility at $25^{\circ} \mathrm{C}$, with a $12 \mathrm{~h} / 12 \mathrm{~h}$ light/dark cycle, and provided with free access to food and water. All experiments were approved and conducted in accordance with the guidelines of the Ethics Committee on Research and Animal Experimentation.

\section{Preparation of Arctium Lappa Extract}

A. lappa plant extract was obtained from $100 \mathrm{~g}$ of ground dry bark dissolved in 1 liter of $70 \%$ ethanol by maceration. A dark flask conditioned to room temperature was used for $72 \mathrm{~h}$, with daily shaking, and was then filtered on filter paper. The filtrate was dried using a rotary evaporator (Eppendorf Vacuum Concentrator Plus, Hamburg, Germany). The dried extract was suspended in 997 $\mu \mathrm{L}$ PBS (phosphate-buffered saline) and $3 \mu \mathrm{L}$ of DMSO (Dimethyl sulfoxide, Sigma-Aldrich, Saint Louis, MO, USA), until a final concentration of $1 \mathrm{~g} / \mathrm{mL}$ was obtained. This extract was filtered and stored in $500 \mu \mathrm{L}$ aliquots at $-20^{\circ} \mathrm{C}$.

\section{Determination of Arctium Lappa Toxicity}

To evaluate the toxicity of the A. lappa extract, we inoculated $300 \mu \mathrm{l}$ of the extract into the peritoneal cavity of C57BL/6 mice at different concentrations $(0.5,5.0$, or $50.0 \mathrm{mg} / \mathrm{kg}$ ) in order to choose only the non-toxic dose for the treatment of the tumor. These doses were chosen based on literature data ((references here) and the Organization for Economic Cooperation and Development (OECD) test method-TG 423 423. Each group was inoculated every 5 days for a total of 25 days and the animal's well-being and survival rate were recorded. The following parameters were observed daily: changes in skin, hair, eyes, mucous membranes, respiration, and central nervous system (tremors, convulsion and sedation) and autonomic nervous system abnormalities (tearing, salivation). After 25 days of the first inoculation, the animals were euthanatized in a $\mathrm{CO}_{2}$ chamber and total and differential counts of peritoneal lavage and blood were examined for the occurrence of an inflammatory reaction. After analyzing the results, the dose of $5 \mathrm{mg} / \mathrm{kg}$ was chosen based on higher dose with lower side-effects.

\section{Cell Culture}

B16F10 cells derived from C57BL/6 mice were cultured in Ham's F10 media at pH 6.9 supplemented with 10\% FBS (fetal bovine serum) and $10 \mu \mathrm{g} / \mathrm{mL}$ gentamicin. All experiments were performed at $80-90 \%$ confluency.

\section{Tumor Implantation}

Primary tumor studies were performed using B16F10 melanoma cells. Tumors were induced by injecting approximately 300,000 cells in $100 \mu \mathrm{L}$ of PBS subcutaneously into the dorsal superior region of mice weighing $\sim 25 \mathrm{~g}$.

\section{Experimental Design}

Animals were divided in four groups: 1) Control Group receiving PBS buffer $(n=5) ; 2)$ receiving plant extract $(\operatorname{Tr})+$ PBS buffer $(n=5)$; 3 ) Tumor (T) + PBS buffer $(n=5)$; and 4) $T$ + plant extract $(\mathrm{T}+\mathrm{Tr})(\mathrm{n}=5)$. Groups 2 and 4 were injected with the plant extract on days $0,5,10,15$ and 20 after inoculation of the tumor (group 4) or PBS injection (group 2). Tumor growth was monitored every 5 days after implantation of B16-F10 melanoma cells, with its dimensions reported as $\mathrm{mm}$ with the aid of a pachymeter. Body weights were monitored daily throughout the experiment. At day 25 , all animals from each group were euthanized by intraperitoneal injection of Ketamine (80 mg/kg) and xylazine $15 \mathrm{mg} / \mathrm{kg}$ ) followed by cervical dislocation. 


\section{Histopathological Analysis}

Tumor and adjacent tissues were collected from all animals and analyzed for skin ulcer formation and tumor size. Lungs, liver, skin and heart were also collected and processed for histopathological analysis. The tissues were immersed in $10 \%$ formaldehyde and embedded in paraffin. Sections of $5 \mu \mathrm{m}$ thick were cut and stained with hematoxilin and eosin (H\&E).

\section{Morphometric Analysis}

Morphometric analysis was performed using an Aristoplan microscope (Leitz, Germany) coupled to a color camera (Leica DFC280, Heerbrugg, Switzerland) and a computer. Mitoses and number of vessels were counted in 15 randomly noncoincident fields at a magnification of $\times 400$.

\section{Statistical Analysis}

Data are expressed as mean \pm SEM. Differences between groups were compared using one-way analysis of variance (ANOVA) and the Newman-Keuls post-hoc test. For differences between two groups, the Student $t$ test was used. A 5\% level of significance was chosen to denote significant differences between the means. Results were analyzed using GraphPad Prism 5.0 (GraphPad Software, San Diego, CA).

\section{Results}

\section{Tumorigenesis}

$\mathrm{T}+\mathrm{PBS}$ group presented a larger melanoma growth rate than the $\mathrm{T}+\mathrm{Tr}$ group (Figure 1). We observed that tumor growth in the $\mathrm{T}+\mathrm{Tr}$ groups was markedly attenuated compared to tumor growth in $\mathrm{T}+\mathrm{PBS}$ group (Figure 1). In $\mathrm{T}+\mathrm{PBS}$ group, the tumors reached maximum width of 22.5 millimeters on the last day of treatment while in $\mathrm{T}+\mathrm{Tr}$ group the tumors' maximum width reached only an average of 12.6 millimeters $(p<0.05)$ (Figure 1). During the treatment period we observed three deaths, two in the T+PBS group and one in the $\mathrm{T}+\mathrm{Tr}$ group. These animals presented weight loss, hypoactivity, larger ulceration of the tumors and increased aggressiveness. Histological analysis of the skin above and around tumor area did not reveal significant architecture alterations. We evaluated histological slices from epidermis, dermis and hypodermis.
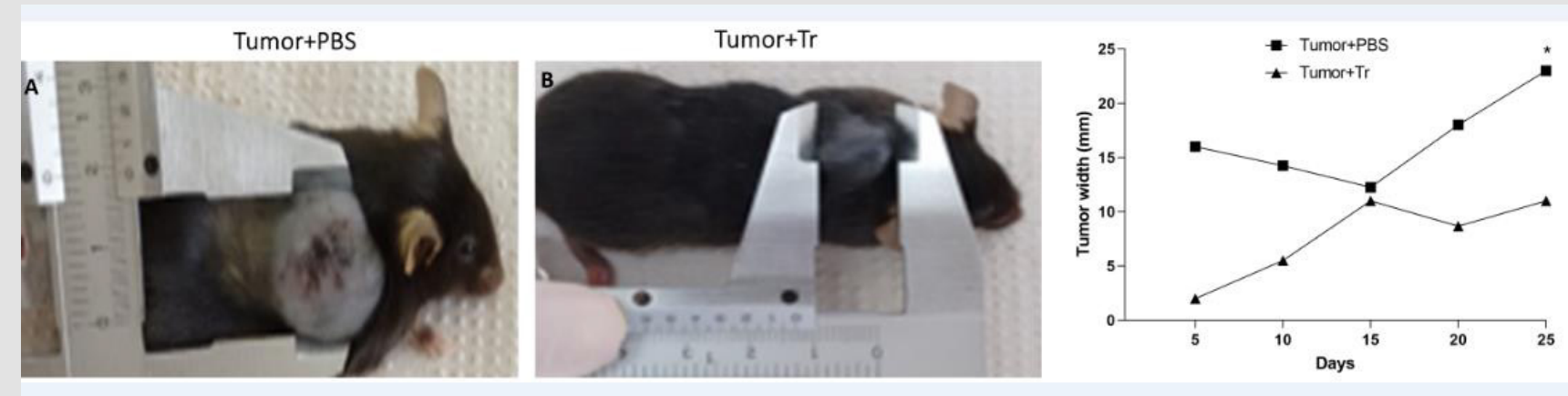

Figure 1: Representative images of C57BL/ 6 mice from

A. $T+P B S$

B. $\mathrm{T}+\mathrm{Tr}$ groups. Representative graph of tumor width in both groups

C. (C)For assessment of tumor size, B16F10 cells were injected in mice treated with Arctium lappa extract $(\mathrm{T}+\mathrm{Tr})$ or PBS

$(\mathrm{T}+\mathrm{PBS})$ every 5 days for 25 days. Tumor width was measured with pachymeter. Data denote mean \pm SEM. * indicates $\mathrm{p}<005$ compared to $\mathrm{T}+\mathrm{PBS}$ group.

\section{Angiogenesis}

Angiogenesis is an important process for the supply of oxygen and nutrients to growing tumors. Fast-multiplying cancer cells require greater demand for oxygen and nutrients than other cells of the body. In the present study, we found that treatment with A. lappa reduced angiogenesis in the tumors of $\mathrm{T}+\mathrm{Tr}$ mice when compared to $\mathrm{T}+\mathrm{PBS}$ group (Figure 2).

\section{Cellular Proliferation}

The mitotic index is a prognostic factor used to classify tumor aggressiveness. We found reduced mitotic cells in tumors from $\mathrm{T}+\mathrm{Tr}$ mice compared to tumors from T+PBS mice (Figure 3), suggesting potentially higher aggressiveness of tumors from PBS-treated mice versus tumors from mice treated with A. lappa. 

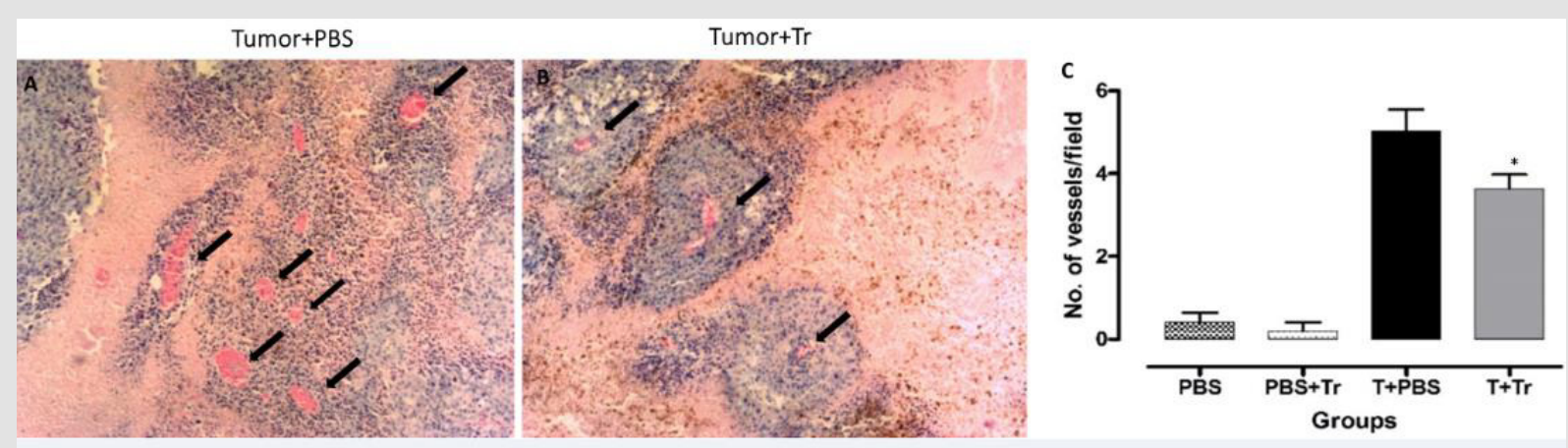

Figure 2: Representative images from histological analysis in H\&E stain showing vessels (black arrows) from

A. $T+P B S$ group.

B. $T+\operatorname{Tr}$ groups, (magnification $40 \times$ )

C. quantification of vessel number per field in tumors from all 4 groups. Data denote mean $\pm \mathrm{SEM}$. * indicates $\mathrm{p}<0.05$ compared to $\mathrm{T}+\mathrm{PBS}$.
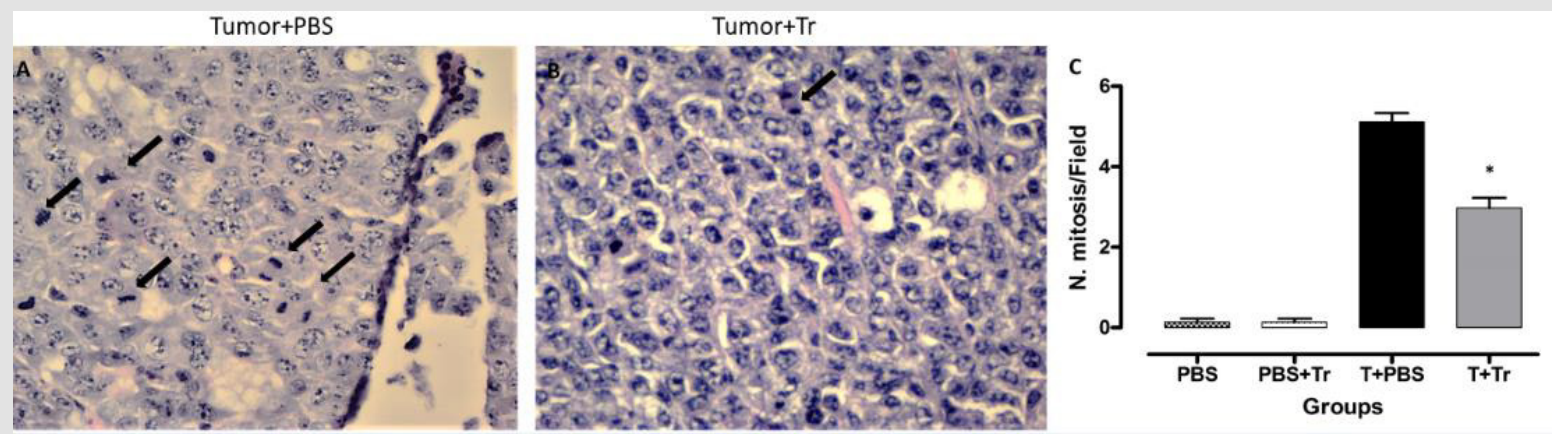

Figure 3: Representative images from histological analysis showing mitotic cells from

A. T+PBS group

B. $T+\operatorname{Tr}$ groups, (magnification $100 \times$ )

C. quantification of the number of cells in mitosis in tumors from all groups. Data denote mean \pm SEM. ${ }^{*}$ indicates $p<0.05$ compared to T+PBS.

\section{Discussion}

In this study, we examined the potential therapeutic action of A. lappa extract against malignant melanoma in mice. A. lappa extract has been demonstrated to exert protection against other cancer-associated pathologies $[13,15,16,17]$, but its effectiveness against melanoma tumors in vivo is still unclear. We found that $A$. lappa extract also exerted a tumorigenic suppressant action in our melanoma model, as evidenced by reduced mitosis in tumorigenic cells and less angiogenesis in tumors from treated animals compared to PBS alone. These observations corroborate previous studies showing anticancer effects of lignans from plant extracts in prostate hyperplasia [15], leukemic cells16 and in the cellular lineages of pancreatic cancer [17] by inhibition of tumor growth of these strains of cancer cells. Previous studies demonstrated that A. lappa regulates immune cell migration and activation, which correlates with favorable outcomes in mouse models of acute inflammation and melanoma progression. These data showed that tumor implantation induced significant accumulation of total leukocytes and neutrophils in the peritoneal cavity, which was suppressed by A. lappa treatment [18]. Arctigenin, one of the lignans extracted from A. lappa, has cytotoxic actions that appear to be specific for cancerous cells, inhibiting their growth and consequently promoting apoptosis, while sparing normal noncancerous cells [13]. Awale et al. [17]. demonstrated that arctigenin has preferential cytotoxicity against nutrient deficient cancer cells due to arctigenin's ability to eliminate the inherent resistance of these cells to energy deprivation. The authors investigated the potential mechanism of action of arctigenin and demonstrated that the compound blocked the activation of Akt, which plays an important role in growth and development of cancer cells [17].

Here we demonstrated that $A$. lappa extract reduced angiogenesis, thus decreasing oxygen and nutrient delivery for melanocytic tumor cells and reducing their ability to maintain high mitotic rate. This finding highlights a novel potentially beneficial 
effect of A. lappa extract in melanoma cancer and suggests that the mechanism of action may be related to nutrient deprivation of tumorigenic cells $\mathrm{Gu}$ et al. [14]. evaluated the citotoxicity of arctigenin in A549 cancer cells and demonstrated that arctigenin promoted the death of tumor cells undergoing glucose deficiency by inhibiting mitochondrial respiration and raising reactive oxygen species concentration in these cells [14]. Additional studies are needed to test whether a similar mechanism is responsible for the anti-tumorigenic actions of $A$. lappa extract observed in the present study Huang et al. [19]. Also reported a decrease of cancer cells in a prostate cancer model by the action of arctinin, another lignan. Arctinin has been shown to promote detachment of cancer cells in culture dishes [19]. The mechanism for cellular detachment process by arctinin appears to involve increased expression of MUC1 , a protein that inhibits cell-cell interactions by steric hindrance of binding sites. Although we did not evaluate the impact of $A$. lappa extract on cell-cell interaction in our melanoma model, it is possible that our treatment may have altered cell-cell interaction to disrupt normal tumor growth and angiogenesis. Another target for therapeutic interventions to treat cancer is to interfere in cellular replication process of tumorigenic cells. Lappaol $\mathrm{F}$, another lignan extracted from A. lappa, induces stagnation of the cellular cycle in G1 or G2 stages, suppressing tumoral cell growth in colon, lungs, uterus lap, prostate, osteosarcoma, and leukemia [20]. Our finding that treatment with A. lappa extract reduced mitotic cell number in mice with melanoma cancer corroborates these previous observations and suggests that a similar stagnation of melanoma cells in G1 or G2 stages may also occur during treatment with $A$. lappa extract.

Cutaneous melanoma is a tumor famous for its ability to metastasize. About $30 \%$ of patients with cutaneous melanoma will present spreading of tumorigenic cells in other tissues [21], mainly hematogenic tissues, but also for lymphatic road and for proximity. A recent study by Lou et al. [22] analyzed the effects of Arctigenin on migration and tumor invasion in breast adenocarcinoma cell lines. The results demonstrated that this lignan significantly inhibited migration and invasion of MDA-MB-231 breast cancer cells due to down-regulation of metalloproteinases (MMP)-2, MMP-9 and heparanase expression [22]. MMP-2 and MMP-9 are extracellular matrix metalloproteinases (gelatinases) that degrade mainly type IV collagen, a fundamental component of the basement membrane. This inhibition facilitates the process of stromal invasion and invasion of blood vessels, which are necessary steps for metastasis. Heparanase is an enzyme that acts both on the cell surface and in the extracellular matrix to degrade heparan sulfate molecules, the main constituent of the endothelial cell layer. The degradation of this component facilitates the penetration of tumor cells into blood vessels and is important for metastasis Pereira et al. [23]. Highlighted the important relationship of MMPs in the development of squamous cell carcinoma, alluding to the possibility of using
MMPs inhibiting agents that could indirectly act as antimetastatic drugs in combating not only epidermoid carcinoma, but perhaps melanoma as well. In the present study, metastases were not observed in any of the organs and tissues analyzed, including heart, lung, skin and liver. However, we did not perform molecular examination to specifically determine if $A$. lappa extract protects against melanoma metastasis.

\section{Conclusion}

Our results suggest that Arctium lappa may represent a potential option as novel therapy for cutaneous melanoma cancer treatment. Our findings demonstrate a beneficial effect of $A$. lappa extract to reduce melanoma tumor growth, which was associated with reduced angiogenesis and mitotic rate. Studies have showed that arctigenin and other lignans extracted from A. lappa may represent a therapeutic strategy with minimum adverse effects on normal tissues. However, larger, more comprehensive studies are needed to better elucidate the tumorigenic suppressant mechanisms of plant extracts in the treatment of cutaneous melanoma.

\section{Funding}

The research was funded by Centro Universitário Barão de Mauáıs intramural faculty grant.

\section{Acknowledgements}

We thank the Adair Ferreira de Lima for the preparation of the slides.

\section{References}

1. Ford D, Bliss JM, Swerdlow AJ, Swerdlow Bruce K, Armstrong, et al. (1995) Risk of cutaneous melanoma associated with a family history of the disease. Int J Cancer 62(4): 377-381.

2. Lew RA, Sober AJ, Cook N, Marvell RMS, Fitzpatrick TB, et al. (1983) Sun exposure habits in patients with cutaneous melanoma: a case control study. J Dermatol Surg Oncol 9(12): 981-986.

3. Maria AG, Dillenburg-Pilla P, Reis IR (2016) Host kinin B1 receptor plays a protective role against melanoma progression. Sci Rep 6: 22078.

4. Lens MB, Dawes M (2004) Global perspectives of contemporary epidemiological trends of cutaneous malignant melanoma. Br J Dermatol 150(2): 179-185.

5. Winsey SL, Haldar NA, Marsh HP, Mike Bunce, Sara E. Marshall, et al. (2000) A variant within the DNA repair gene XRCC3 is associated with the development of melanoma skin cancer. Cancer Res 60(20): 56125616.

6. International agency for research on cancer. Melanoma of skin.

7. Rastogi RP, Richa, Kumar A, Tyagi MB, Sinha RP, et al. (2010) Molecular mechanisms of ultraviolet radiation-induced DNA damage and repair. J Nucleic Acids 2010: 592980.

8. Holman, CDAJ, BK PJ (1986) Relationship of cutaneous malignant melanoma to individual sunlight-exposure habits. J Natl Cancer Inst 76(3): 403-414.

9. Kim J, Lazar AJ, Davies MA, Jade Homsi, Nicholas E Papadopoulos, et al. (2012) BRAF, NRAS and KIT sequencing analysis of spindle cell melanoma. J Cutan Pathol 39(9): 821-825. 
10. Soares de Sá BC, Moredo LF, Gomes EE, de Araújo ESS, Duprat JP, et al. (2018) Hereditary melanoma: a five-year study of Brazilian patients in a cancer referral center - phenotypic characteristics of probands and pathological features of primary tumors. An Bras Dermatol 93(3): 337 340.

11.Lima AR, Barbosa VC, Santos PRF, Gouvêa CMCP (2006) In vitro evaluation of the antioxidant activity of the hydroalcoholic extract of burdock leaves. Rev Bras Farmacogn 16(4): 531-536.

12. Yao X, Zhu F, Zhao Z, Liu C, Luo L, et al. (2011) Arctigenin enhances chemosensitivity of cancer cells to cisplatin through inhibition of the STAT3 signaling pathway. J Cell Biochem 112(10): 2837-2849.

13.Susanti S, Iwasaki H, Itokazu Y, Mariko Nago, Naoyuki Taira, et al. (2012) Tumor specific cytotoxicity of arctigenin isolated from herbal plant Arctium lappa L. J Nat Med 66(4): 614-621.

14.Gu Y, Qi C, Sun X, Ma X, Zhang H, et al. (2012) Arctigenin preferentially induces tumor cell death under glucose deprivation by inhibiting cellular energy metabolism. Biochem Pharmacol 84(4): 468-476.

15.Ming DS, Guns E, Eberding A, Towers GH (2004) Isolation and characterization of compounds with anti-prostate cancer activity from Arctium lappa L. using bioactivity-guided fractionation. Pharm biol 42(1): 44-48.

16. Matsumoto T, Hosono-Nishiyama K, Yamada H (2006) Antiproliferative and apoptotic effects of butyrolactone lignans from Arctium lappa on leukemic cells. Planta Med 72(3): 276-278.

\section{ISSN: 2574-1241}

DOI: 10.26717/BJSTR.2021.34.005581

Tefé Silva C. Biomed J Sci \& Tech Res

(C) This work is licensed under Creative

Submission Link: https://biomedres.us/submit-manuscript.php
17. Awale S, Lu J, Kalauni SK, Kalauni, Yukiko Kurashima, et al. (2006) Identification of arctigenin as an antitumor agent having the ability to eliminate the tolerance of cancer cells to nutrient starvation. Cancer Res 66(3): 1751-1757.

18. Nascimento BAC, Gardinassi LG, Silveira IMG, Marília G Gallucci, Mariana A Tomé, et al. (2019) Arctium lappa extract suppresses inflammation and inhibits melanoma progression. Medicines 6(3): 81.

19. Huang DM, Guh JH, Chueh SC, Teng CM (2004) Modulation of antiadhesion molecule MUC-1 is associated with arctiin-induced growth inhibition in PC-3 cells. Prostate 59(3): 260-267.

20.Sun Q, Liu K, Shen X, Lappaol F, Hu Y, et al. (2013) a novel anticancer agent isolated from plant Arctium Lappa L. Mol Cancer Ther 13(1): 4959.

21. Allen BC, Mohammed TL, Tan CD, Miller DV, Williamson EE, et al. (2012) Metastatic melanoma to the heart. Curr Probl diagn radiol 41(5): 159164.

22.Lou C, Zhu Z, Zhao Y, Zhu R, Zhao H, et al. (2017) Arctigenin, a lignan from Arctium lappa L., inhibits metastasis of human breast cancer cells through the downregulation of MMP-2/-9 and heparanase in MDAMB-231 cells. Oncol Rep 37(1): 179-184.

23. Pereira AC, Carmo ED, Silveira VAS, Amadei SU, Rosa LEB, et al. (2006) The role of MMP-2 and -9 in the development of squamous cell carcinoma. Rev Bras Cancerol 52(3): 257-262.

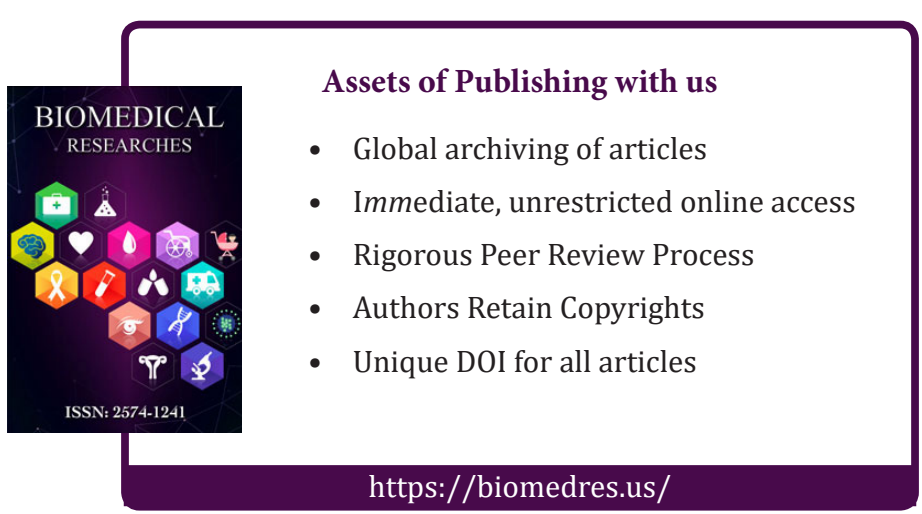

